\title{
Central Corneal Thickness - A Must In Management of Glaucoma
}

\author{
Dr Sankalp Seth ${ }^{1}$, Dr Gaurav Kapoor ${ }^{2}$, Dr Vijay Mathur ${ }^{3}$, Dr Deepti Sahran ${ }^{4}$ \\ ${ }^{1}$ Classified Specialist (Ophthalmology) Military Hospital Allahabad, \\ ${ }^{2}$ Associate Professor (Ophthalmology) ACMS \& Base Hospital Delhi Cantt, \\ ${ }^{3}$ Professor (Ophthalmology) Command Hospital Chandigarh, \\ ${ }^{4}$ Resident Administrator, Dept Of Hospital Administration, AIIMS New Delhi.
}

\begin{abstract}
:
Purpose: To determine the correlation of central corneal thickness (CCT) with intraocular pressure measurement (IOP) \& to reclassify the patients of Primary Open Angle Glaucoma (POAG), Normal Tension Glaucoma (NTG) \& Ocular Hypertension (OHT) based on their corrected IOP.

Design: Retrospective analysis.

Material \& Methods: It was a retrospective non-interventional, observational study. 100 cases \& 20 controls were inducted in the study. Central corneal thickness was measured by ultrasonic pachymeter. Corrected IOP was calculated based on CCT value (correction factor of $3.0 \mathrm{~mm} \mathrm{Hg}$ for every $50 \mu \mathrm{m}$ of difference from $550 \mu \mathrm{m}$ ) using a linear formula Corrected IOP $=$ Measured IOP $-(C C T-550) / 50 \times 3.0 \mathrm{~mm} \mathrm{Hg}$.

Results: Total of 100 cases (POAG -54, NTG- 26 \& OHT-20) \& 20 controls were included in the study. There was no significant difference in CCT between controls and patients with POAG but the CCT in the group with $N T G$ was significantly lower than that in the control group or the group with POAG $(P<.001)$, and the CCT in the group with OHT was significantly higher than in controls or patients with POAG $(P<.000)$. There was significant difference in IOP values in NTG \& OHT cases when CCT correction was taken in measuring the IOP ( $p$ value $<0.000)$

Conclusion: Patients with NTG have a thinner CCT than do patients with POAG or controls. Correcting IOP for corneal thickness, $42 \%$ of the patients with NTG could be reclassified as having POAG, and $30 \%$ of the patients with OHT as normal and $13 \%$ of POAG cases were actually found to be cases of NTG.

Keywords- IOP (Intraocular pressure)

CCT (Central corneal thickness)

POAG (Primary open angle glaucoma)

OHT (Ocular hypertension)

NTG (Normal tension glaucoma)
\end{abstract}

\section{Introduction}

Glaucoma is a neurodegenerative disorder of the optic nerve and one of the leading causes of irreversible blindness worldwide. ${ }^{1}$ It is characterized by progressive atrophy of the optic nerve head secondary to the loss of optic nerve fibres. The loss of ganglion cells is irreversible nevertheless in nearly all the cases blindness is preventable. This prevention requires early detection and proper treatment. Early diagnosis of the disease is directly linked with a favourable prognosis and lesser disability. The major risk factors for glaucoma include intraocular pressure, age, race, family history, myopia and central corneal thickness. The most important risk factor for glaucoma is elevated intraocular pressure (IOP) making its accurate measurement essential for disease detection and patient follow up. ${ }^{2-3}$ To get accurate IOP readings, the measurements should be adjusted for Central corneal thickness (CCT). ${ }^{4}$ However, the incorporation of CCT-adjusted IOP measurements into daily clinical practice was limited until recently, when the Ocular Hypertension Treatment Study ${ }^{5}$ reported that CCT was a strong predictor for the development of primary open-angle glaucoma (POAG) in patients with ocular hypertension(OHT). Moreover, individuals with CCT's of $555 \mu \mathrm{m}$ or less had a 3 times greater risk of developing glaucoma compared with patients with CCT's of greater than $588 \mu \mathrm{m}$.

Furthermore, variations in mean CCT have been observed in patients with different types of glaucoma. Intraocular pressure measured may be overestimated or underestimated in thick or thin corneas, respectively When the CCT is not considered, there are chances of misdiagnosis as patients with normal-tension glaucoma have thinner corneas and those diagnosed as glaucoma suspect have thicker corneas. ${ }^{6}$ Performing pachymetry greatly influences the management of all patients as it allows the clinicians to pursue less aggressive treatment of patients with pseudo-ocular hypertension, setting the real target intraocular pressure in patients with glaucoma, and in detecting an elevated intraocular pressure in otherwise normal patients with thin corneas. ${ }^{7}$ 
While Goldmann applanation tonometry is the preferred method of measurement of IOP, several factors, including corneal thickness, influences its accuracy. Goldmann calibrated the applanation tonometer for a central corneal thickness of $520 \mu \mathrm{m}$. There is a lack of general agreement regarding the correction factor that should be used for adjusting the IOP, measured by Goldmann tonometry. Correction Factors seen in literature have ranged from approximately 1.00 to $3.57 \mathrm{~mm} \mathrm{Hg}$ for every $50-\mu \mathrm{m}$ deviation.

Central corneal thickness has been reported to be higher in patients with ocular hypertension (OHT) and lower in patients with normal tension glaucoma (NTG), as compared with patients with primary open-angle glaucoma (POAG) and healthy patients. These discrepancies may cause misdiagnoses in some cases because of an artefact of intraocular pressure (IOP) measurement. This study was undertaken to measure the correlation of central corneal thickness with measurement of Intraocular pressure, to study \& compare the central corneal thickness in patients of glaucoma \& glaucoma suspects $\&$ and furthermore to determine the potential number of patients with glaucoma (POAG) misdiagnosed as having NTG and vice versa or normal persons being misdiagnosed as OHT due to variations in corneal thickness

\section{Materials \& Methods}

The study was conducted in the ophthalmology OPD of an army hospital. It was a retrospective observational study. 100 cases (POAG, NTG,OHT) \& 20 controls were inducted in the study. Patients of POAG (IOP> $21 \mathrm{mmHg}$ in the presence of a typical glaucomatous disc and field changes and an open angle on gonioscopy), NTG (IOP $<21 \mathrm{mmHg}$ with typical glaucomatous disc and field changes and open angle on gonioscopy), OHT (IOP> $22 \mathrm{mmHg}$ or higher with normal disc, normal visual fields and open angle on gonioscopy) were included in this study. Exclusion criteria consisted of patients with secondary glaucoma, patients with corneal pathology like corneal ulcers, keratoconus etc and patients who had undergone refractive surgery. Type of glaucoma \& number of medications being taken were recorded from their medical records. All patients underwent a extensive ophthalmological examination including visual acuity by Snellen visual acuity chart, IOP measurement by Goldmann applanation tonometer, visual field analysis by 30-2 static threshold perimetry, assessment of anterior chamber angle by gonioscopy, and optic nerve head examination including vertical \& horizontal cup-disc ratio by direct ophthalmoscopy. Central corneal thickness was measured by ultrasonic pachymeter. Corrected IOP was calculated based on CCT value (correction factor of $3.0 \mathrm{~mm} \mathrm{Hg}$ for every $50 \mu \mathrm{m}$ of difference from $550 \mu \mathrm{m})$ using a linear formula ${ }^{\mathbf{1 0}}$ Corrected IOP $=$ Measured IOP $-(C C T-$ $550) / 50 \times 3.0 \mathrm{~mm} \mathrm{Hg}$. In case of bilateral disease, poor eye parameters was considered. However in symmetrical disease, right eye parameters were taken. In controls right eye parameters were incorporated in the study. Finally the means of data \& standard deviation was calculated using Microsoft excel software and analyzed by using Epi 2002. A p value of $<0.05$ was taken as significant.

\section{Results}

A total of 100 cases (POAG -54, NTG- 26 \& OHT- 20) \& 20 controls were included in the study. There was no significant difference in the mean age group of patients. $63 \%$ of total cases were males \& $37 \%$ of cases were females. In the control group, equal distribution of gender was done, $50 \%$ males $\&$ females each. There was notable difference in gender distribution in cases with male predominance in POAG \& OHT and female predominance in cases of NTG .(Table 1)

Table 1 Gender Distribution of cases (POAG, NTG, and OHT)

\begin{tabular}{|l|l|l|l|}
\hline Disease & Males & Females & Total \\
\hline POAG & $40(74 \%)$ & $14(26 \%)$ & 54 \\
\hline NTG & $9(34 \%)$ & $17(66 \%)$ & 26 \\
\hline OHT & $14(70 \%)$ & $6(30 \%)$ & 20 \\
\hline Total (Gender wise) & $63(63 \%)$ & $37(37 \%)$ & 100 \\
\hline
\end{tabular}

There was no significant difference in CCT between controls $(554.25 \pm 28.18 \mu \mathrm{m})$ and patients with POAG $(549.77 \pm 25.38 \mu \mathrm{m})$, but the CCT in the group with NTG $(517.65 \pm 39.88 \mu \mathrm{m})$ was significantly lower than that in the control group or the group with POAG $(\mathrm{P}<0.001)$, and the CCT in the group with OHT $(591.4 \pm 37.79 \mu \mathrm{m})$ was significantly higher than in controls or patients with POAG $(\mathrm{P}<0.000)$ (Table 2$)$

Table 2 Mean CCT of cases (POAG, NTG, and OHT) \& controls

\begin{tabular}{|l|l|l|l|l|}
\hline Cases & POAG & NTG & OHT & CONTROLS \\
\hline Mean CCT & $549.77 \mu \mathrm{m}$ & $517.65 \mu \mathrm{m}$ & $591.40 \mu \mathrm{m}$ & $544.25 \mu \mathrm{m}$ \\
\hline
\end{tabular}

The mean CCT values of POAG group \& Controls were almost in similar range but mean CCT of NTG was lower than that of POAG, OHT \& controls \& mean CCT of OHT group was higher than rest of the cases (POAG, NTG) \& controls. 
There was significant difference in IOP values in NTG \& OHT cases when CCT correction was taken in measuring the IOP ( $\mathrm{p}$ value $<0.000$ ). Using the correction for corneal thickness, IOP in patients with NTG was underestimated by a mean of $2.0 \mathrm{~mm} \mathrm{Hg}$ IOP in patients with OHT was overestimated by a mean of $2.42 \mathrm{~mm}$ $\mathrm{Hg}$ (Table 3 \& 4).

Table 3 Comparison of mean IOP values in NTG group before \& after CCT correction

\begin{tabular}{|l|l|l|l|}
\hline CASES(NTG) & Mean $(\mathbf{m m H g})$ & SD & SE \\
\hline IOP without CCT correction & 18.17 & 1.6 & 0.329 \\
\hline IOP corrected for CCT & 20.17 & 2.48 & 0.487 \\
\hline
\end{tabular}

A paired t-test was used which showed a significant difference.

$\mathrm{N}=26$ pairs, $\mathrm{t}-\mathrm{value}=4.28$ and $\mathrm{p}$ value of $0.000(<0.05)$ was noted .

Table 4 Comparison of mean IOP values in OHT group before \& after CCT correction

\begin{tabular}{|l|l|l|l|}
\hline Cases(OHT) & Mean(mmHg) & SD & SE \\
\hline IOP Without CCT Correction & 25.62 & 3.35 & 0.749 \\
\hline IOP Corrected For CCT & 23.16 & 3.13 & 0.700 \\
\hline
\end{tabular}

A paired t-test was used which showed a significant difference.

$\mathrm{N}=20$ pairs, $\mathrm{t}$-value $=4.78$ and $\mathrm{p}$ value of $0.000(<0.05)$ was noted.

Correcting IOP for corneal thickness, $42 \%$ of the patients with NTG could be reclassified as having POAG, and $30 \%$ of the patients with OHT as normal and $13 \%$ of POAG cases were actually found to be cases of NTG.

\section{Discussion}

Glaucoma is the leading cause of irreversible but preventable blindness in the world. Being a preventable cause it is extremely important to timely and accurately diagnose the disease at the earliest and to start the necessary treatment.Three main factors are taken into consideration for monitoring a case of glaucoma which includes Optic nerve head changes, Field defects and IOP measurements. Hence accurate IOP measurement (With incorporation of correction factor for CCT) is a must in treatment of a case of Glaucoma.

In this study patients with NTG had considerably thinner CCT than did the group with POAG or the controls, while the group with OHT had notably thicker CCT than did patients with POAG/NTG or controls which corroborates with various other studies. ${ }^{8-10}$ Underestimation of the IOP in patients with POAG who have thin corneas may lead to a misdiagnosis of NTG, while overestimation of the IOP in normal subjects who have thick corneas may lead to a misdiagnosis of OHT. Similarily patients of NTG with thin cornea may actually be cases of POAG when CCT corrected IOP is taken. The effect of central corneal thickness on Goldmann applanation tonometry accuracy appears to be significant and clinically relevant especially in cases of OHT \& NTG.

In the study done by Ravi Thomas et $\mathrm{al}^{10}$ when corneal thickness was taken into account (31\%) of their patients thought to have NTG actually met the criteria for a diagnosis of POAG. Similarly, many patients diagnosed as having OHT (25 [56\%] in their study) actually had normal IOP.

In this study when corneal thickness was taken into account, $42 \%$ of patients of NTG actually met the criteria for a diagnosis of POAG. Similarly, many patients diagnosed as having OHT (30\% of OHT cases in this study) actually had normal IOP. 13\% of POAG cases actually had NTG. One third of the patients diagnosed as having OHT, but ultimately found to have normal IOP, had been undergoing treatment with one or more medications.

With the increasing number of patients with iatrogenically thinned cornea by laser refractive surgery, the measured IOP in these patients is lower than the true value. Most individuals who undergo laser refractive surgery are myopic who are at a 2-6 fold increased risk of developing glaucoma, the risk being proportionate to the degree of myopia. This is of particular concern with higher myopic corrections in which laser refractive surgery is associated with more severe corneal thinning and greater IOP underestimation.

Limitation of this study included the small sample size of population, there is a need to study the correlation in a large indian population based study to know the real impact. Secondly the CCT was measured with ultrasonic pachymeter which is only one of the means of measuring CCT. Studies ${ }^{11}$ have shown that, depending on the method used, statistically different CCTs may be obtained. Optical low coherence reflectometry represents the most precise pachymetric method available, with its measurements reproducible to $1 \mu \mathrm{m} .{ }^{12}$ In the future, appropriate refinement of the correction formula may need to be undertaken, depending on the corneal pachymetric method adopted.

The routine measurement of CCT is critical for the proper management $\&$ follow up of patients with glaucoma and glaucoma suspect not only for cost reasons, but most important, for effective therapy. 


\section{References}

[1]. McKinnon SJ. Glaucoma: Ocular Alzheimer's disease. Front Biosci. 2003;1:11401156.

[2]. Wilson MR. Epidemiological features of glaucoma. Int Ophthalmol Clin. 1990;30:153 160.

[3]. Horowitz GS, Byles J, Lee J, D'Este C. Comparison of the Tono-Pen and Goldmann tonometer for measuring intraocular pressure in patients with glaucoma. Clin Exp Ophthalmol 2004;32:584-589.

[4]. Whitacre M, Stein R, Hassanein K. The effect of corneal thickness on factors that predict the onset of primary open-angle glaucoma. Arch Ophthalmol. 2002;120:714-720. applanation tonometry. Am J Ophthalmol. 1993;115:592-596.

[5]. Gordon M, Beiser J, Brandt J, et al. The Ocular Hypertension Treatment Study: baseline Arch Ophthalmol. 2002;120:714-720.

[6]. Chopra V, Verma R, Francis VA, Wu J, Torres M : Los Angeles Latino Eye Study Group . Type II DM \& risk of OAG. Ophthalmolgy.2008Feb;115(2) :227-232.

[7]. Tanaka GH. Corneal pachymetry: a prerequisite for applanation tonometry? Arch Ophthalmol. 1998;116:544-545

[8]. Vinay Nangia, Jost B. Jonas et al Central Corneal Thickness and Its Association with Ocular and General Parameters in Indians: The Central India Eye and Medical Study ophthalmology Volume 117, Issue 4, Pages 705-710 (April 2010)

[9]. Carolyn Y. Shih, MD; Joshua S. Graff Zivin, PhD; Stephen L. Trokel, MD; James C. Tsai, MD Clinical Significance of Central Corneal Thickness in the Management of Glaucoma Arch Ophthalmol. 2004;122:1270-1275

[10]. René-Pierre Copt, MD; Ravi Thomas, MD; André Mermoud, MD Corneal Thickness in Ocular Hypertension, Primary Open-angle Glaucoma, and Normal Tension Glaucoma ArchOphthalmol. 1999;117:14-16.

[11]. Gonzalez-Meijome J, Cervino A, Yebra-Pimentel E, et al. Central and peripheral corneal thickness measurement with Orbscan II and topographical ultrasound pachymetry. J Cataract Refract Surg. 2003;29:125-132.

[12]. Ventura AC, Bohnke M, Mojon DS. Central corneal thickness measurements in patients with normal tension glaucoma, primary open angle glaucoma, pseudoexfoliation glaucoma, or ocular hypertension. Br J Ophthalmol. 2001;85:792-795. 